\title{
Improving Design Productivity and Product Data Consistency
}

\author{
From Design Intent to Solid Models through Technological Links
}

\author{
F. GIGUÈRE ${ }^{1}$, L. RIVEST ${ }^{2}$ and A. DESROCHERS ${ }^{1}$ \\ 1 University of Sherbrooke, Department of Mechanical Engineering; \\ 2) École de technologie supérieure, Department of Automated Production Engineering
}

\begin{abstract}
Computer Aided Design systems still suffer from a number of shortcomings with regards to the full design process: they provide limited solutions for maintaining product data consistency and offer no assistance to relieve designers from tedious, repetitive, solid modeling work. This paper presents a framework for improved support of the design process from preliminary to detailed design phase. The proposed solution aims at managing product data consistency within the assembly model as well as at assisting designers with specific geometric definition tasks by exploiting the design context. First, relationships between parts are captured by establishing specialized technological links, called derivation links, that encapsulate specific design knowledge. These links are then used to apply design knowledge to the design context and to derive design feature geometry, called pseudo-imprints. These links are maintained and later exploited to control design change propagation. An aircraft structure design example illustrates these concepts.
\end{abstract}

Key words: Features, Assembly Modeling, Feature-Based Design, CAD, Modeling Method

\section{INTRODUCTION}

Products rarely consist of a single part. They are usually composed of an array of assembled parts, allowing different materials to be used. Generally, parts of different sizes and shapes are best produced separately. The use of computers in design has allowed modelling these parts as 3D solids and to assemble them virtually before actual manufacture. Computer aided design (CAD) tools have therefore helped reduce the number of errors before the 
manufacturing stage as well as the number of prototypes needed. While they have many advantages, these methods still present several problems. One of these is that modelling complex parts is time consuming even for highly skilled designers, who are required to carry out detailed and tedious tasks. Another problem is the build-up of massive databases containing inconsistent information, as a result of the increasing capability to create multiple versions of electronic documents and the availability of cheap data storage.

With regard to the first problem, the use of feature-based design has improved the situation by allowing designers to work at higher levels of abstraction. Portions of geometry are instanced, sized and located quickly by specifying a minimum set of parameters while the feature modeller completes the details. This modelling method is starting to emerge in commercial CAD software, in which, however, it is usually applied to the design of parts considered individually rather than within the design context.

In terms of the second problem, little has been done so far. Assemblies are typically modelled as a hierarchy of subassemblies and components, but current CAD software captures little of the semantics of relationships between parts. Currently, propagating a modification made to one part of an assembly requires the designer to manually perform a series of tedious operations to update all impacted models accordingly, in order to maintain the global coherence of the assembly model. Relying on such manual interventions easily leads to design errors that are sometimes revealed on the shop floor. The use of assembly features in assembly modelling is a topic of research aiming to capture relationships between parts in order to solve this data consistency problem.

A four-year research project involving Bombardier Aerospace, Université de Sherbrooke and École de Technologie Supérieure was undertaken in 1998 to define an information system architecture that would allow exploiting CAD data with Product Data Management tools. In this paper, we focus on the two problems mentioned above (data consistency and tedious product modelling tasks). A framework is proposed that manages multi-model links to ensure information consistency while increasing the productivity of specific modelling tasks. It allows the capture of design intent by establishing technological links between features belonging to different parts. These links are specialized so as to embed knowledge relevant to specific design tasks. Specialized applications are triggered to assist designers in creating a feature "pseudo-imprint" geometry obtained from a "derivation link". 


\section{RELATED WORK}

In this section, research works falling into the areas of Design processes, Features and Assembly modelling, are briefly reviewed. In the area of design processes, the bottom-up approach has been perceived to be the most widely used for assembly modelling within CAD systems. This approach requires that parts be fully defined before creating the assembly. However, a more natural way is to model assemblies with the top-down approach, which Libardi (Libardi 88) defines as a recursive subdivision of an initial abstract concept into logical sub-assemblies until the part level is reached. This approach allows to better support the design process where the designer thinks in terms of abstract function before being concerned with the geometry (Ullman 97).

Research on assembly modelling has been conducted in various ways with different goals in mind. Several assembly models have been proposed (Rimscha 90) (Sodhi 91) (Mailharro 98) (Bronsvoort 94). Gui and Mäntylä (Gui 93) distinguish themselves by proposing a generic assembly model capable of supporting both conceptual design and detailed design, developing a design case-based reasoning system. They use an assemblymodelling scheme with a function-based approach, "first assembly then components". Therefore they use a top-down process which is not limited to merely designing with features and which is closer to the way designers work. Their proposal aims at taking into account the assembly at the early stage of the design process. In a following paper (Gui 94) they develop these concepts further by presenting a general assembly model that provides a function-oriented view for design knowledge and a module-oriented view for manufacturing knowledge. They state that features are too low-level to support the early phases of design.

Shah and Mäntylä (Shah 95) define a form feature as the generic shape or characteristic of a product with which engineers can associate attributes and knowledge for reasoning about the product. The concept of assembly feature is defined as an association between two form features which belong to different parts. Different approaches to assembly modeling are described and the assembly feature concept is presented with its various advantages:

- Assembly features allow assembly creation at a higher level by storing all of the constraints between the mating features as a single unit.

- Assembly features allow constraints to be expressed in terms of feature dimensions rather than face relations.

- Assembly features allow design changes made to a part to propagate to other parts in an assembly. 
Shah (Shah 92) introduces the notion of feature-based assembly modelling. He developed a system for assembly design which encapsulates different constraints between mating features. His research targets bottom-up assembly modelling in the detail design phase. In a subsequent article (Shah 1993), he explores the advantages and limitations of procedural and declarative approaches for assembly modelling. He explains how some structures can be used to define relationships between assemblies, parts, features, and feature volume primitive. He proposes methods for deriving the location of an assembly unit from high-level constraint specifications.

The goal of the FEAST project (Feature-based Assembly Techniques) (Bordegoni 99) (Deneux 99) was to find a way to extend the use of features from the design area to help solve assembly problems. Bordegoni and Cugini (Bordegoni 99) developed concepts on how assembly features can be used to solve assembly problems, and presented a graphical formalism for representing assembly models and structures. They also introduced a process for knowledge formalization focusing on user/system activity sharing and knowledge sharing. An assembly feature-based demonstrator was created to validate these concepts.

Within the same framework Deneux (Deneux 99) advocates assembly features, explaining how they can be characterized in any field concerned with assembly design. He illustrates the assembly structure with a matrix where the relationships between different part features can be emphasized. $\mathrm{He}$ also proposes a process for formalizing feature data and behaviour. Finally he presents a demonstrator and a prototype of an assembly modeller for the aeronautic industry.

The work described in this paper differs from the above studies in several aspects. Firstly, while the bottom-up approach is the most widely used in the application of assembly features to the detail design phase of assembly modelling (i.e. assembly modelling using fully defined parts), we propose a method closer to the top-down approach. We begin at the preliminary design phase, where parts are only partially defined. These partially defined parts are placed in the design context and we form technological links to assist in the creation of the missing features. As a result, our method captures the design intent right from the preliminary design phase and exploits it well into the detail design phase. Secondly, our method can be used with any $\mathrm{CAD}$ system, rather than with design-by-feature modellers, since the technological links we create between parts do not involve CAD-generated features. Furthermore, we use the technological links (defined further) to capture and reuse design intent and to assist in the creation of certain features. To be useful, these technological links must be strongly typified, meaning that a specific link must be defined for the resolution of a specific design situation. Implementing these concepts of course leads to the 
development of "task specific" applications providing relevant technological links for the problem at hand, thus endorsing the underlying idea that improving design productivity requires creating specialized CAD tools to replace or complement the generic ones currently available on the CAD market. At the time of writing, these concepts are being implemented in an application to illustrate them. We insist, however, that the proposed concepts remain generic in their nature and that they can be applied to a vast collection of design problems. For example, Macabies and other members of our research team used technological links to create an application that helps design fasteners between parts (Macabies 00).

\section{STATEMENT OF WORK}

It was evaluated that $80 \%$ of the time spent on some detail design tasks does not involve creative work (educated guess based on observation and interviews with designers). Consequently, the main objective of this article is to propose a solution for capturing and reusing knowledge in order to maintain data consistency within the assembly model and to improve, assist and automate repetitive and tedious modeling tasks. To be more specific, we wish to:

- Propose a solution to capture design intent right from the preliminary design phase.

- Use the top-down approach by working with assemblies right from the preliminary design phase when parts are not fully defined.

- Exploit the design context (i.e. the virtual environment of the part) and some design rules to assist the designer in the definition of specific form features.

- Ensure information consistency within the digital mock-up.

- Provide assistance to well-defined and repetitive design tasks.

- Develop applications that are not linked to specific CAD systems and that do not require one to work in a feature-based design environment.

\section{CONCEPTUAL ELEMENTS OF THE APPROACH}

This project proposes a framework for developing applications on top of current CAD systems in order to assist designers in defining missing features on parts by capturing and exploiting the design intent. These missing 
features are those whose geometry is strongly constrained by the design context. Hence, when designing a complex product, parts are defined up to a certain point in the design context. They are then linked to other parts of the assembly so that the missing features, whose design depends on the context, may be created with the help of the application. The goal of this approach is first to improve part modelling by facilitating the creation of certain features. Secondly, product data consistency is managed by creating links between related parts so that modifications, such as dimensional changes, can be propagated even after part models have been digitally assembled.

This requires that the application be able to:

- Capture the relationships between related parts by creating a link between them;

- Create a feature using the information derived from the part by the link;

- Preserve this link to allow design change propagation;

To fulfil such requirements, the following concepts will be used: design feature, design context, technological link, derivation link, pseudo-imprint, target features and parts, datum features and parts, assisted creation.

- Design feature: Functional element of an object which has an engineering meaning and that can act as a handle over a set of information (henceforth, we will use the word "feature" to refer to "design feature").

- Design context: The virtual environment in which the designer is working, defined by a portion of the digital mock-up.

- Technological link: A link between two entities that carries technological information and that is strongly typified.

- Derivation link : Specialized technological link that encapsulates a unidirectional relation between a target feature and a datum feature of the design context.

- Pseudo-imprint : Geometry of a target feature on a part determined automatically by applying knowledge to the geometry of another part of the design context by means of a "derivation link".

- Target feature : Instance of a design feature for which a pseudo-imprint is defined.

- Datum feature : Design feature from which the target feature definition is derived.

- Target part : Part on which a target feature is defined and created.

- Datum part : Part to which the datum feature belongs.

- Assisted creation : Automatic construction process over which the user maintains a certain level of control. 


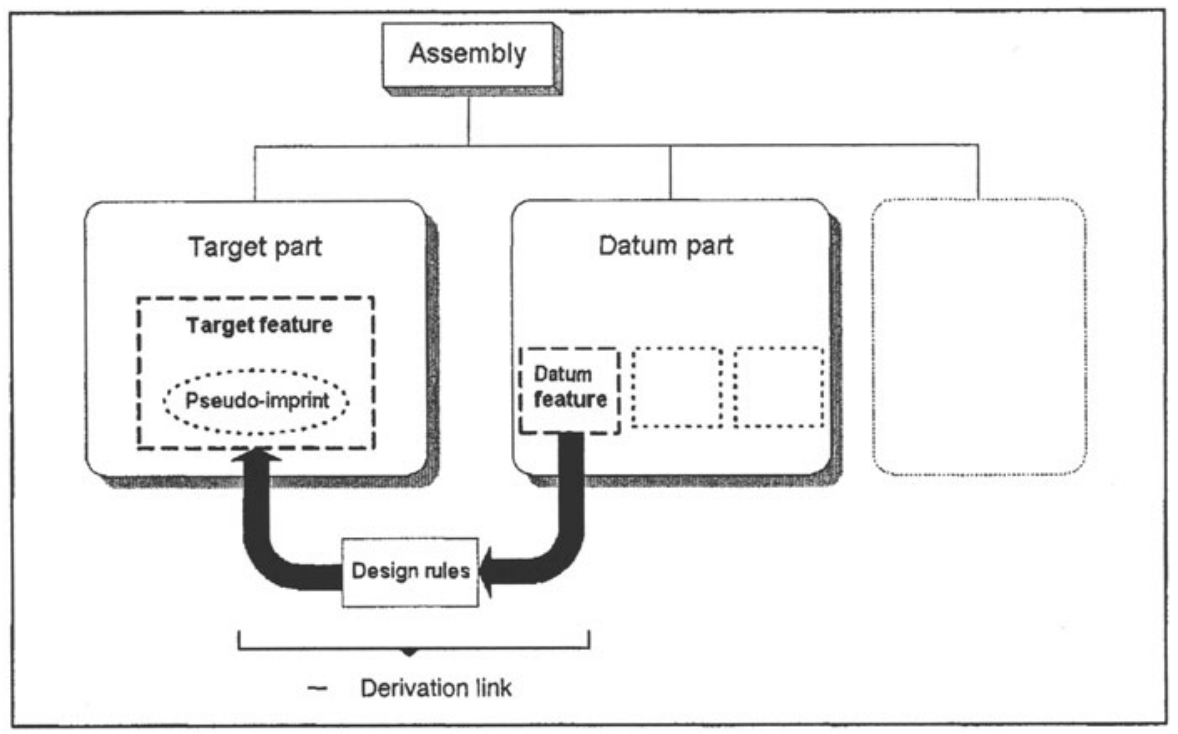

Figure 1. Pseudo-imprint and Derivation link structure

Figure 1 shows a schematic representation of the pseudo-imprint and derivation link concepts. While the "derivation link" may be likened to an assembly feature, unlike the latter it does not relate two pre-existing features. Rather, it relates a datum feature to another part, with the purpose of creating a new feature on the target part, for which the geometrical parameters define a pseudo-imprint. Therefore, the geometry of the derived target feature is a pseudo-imprint of a reference feature on the datum part. This derivation link implies a unidirectional relation. This means that a modification made to the datum part will be propagated to the pseudo-imprint when the geometry of the datum feature changes or when the relative position of both parts is modified.

Generic properties of the target feature are stored in the application. They record the feature's engineering significance and generic shape and provide the basis of its behaviour. These properties allow the shape to be generated by means of pre-defined procedures arising from a set of design rules relevant to a specific problem. Focusing on specific well-defined problems allows us to strongly categorize the derivation link, which in turn improves efficiency by letting us know exactly what we are working with.

The design rules are applied to the independent parameters obtained from the other models in the assembly, i.e., the design context. The technological link established between the datum and the target is used to apply knowledge to these independent parameters and thus derive the geometry of the target 
feature. This is why we say that a pseudo-imprint is the geometry of a target feature defined by the design context.

\section{PROPOSED PROCESS}

As mentioned previously, this project seeks to define general concepts to help improve the productivity of some design tasks while ensuring product data consistency. One of the keys to achieving this goal is to focus on specific design problems that can be solved automatically by applying engineering knowledge through dedicated applications. A task-specific oriented method is thus used to restrict the application domain. In order to implement this method in a design methodology, it is necessary to fully characterize the problem at hand by identifying its specific geometric configuration, the criteria of applicability, the specific design rules involved as well as the design and manufacture constraints.

This type of specialization calls for the use of the procedural approach over the declarative approach. In the procedural approach, generic features are pre-defined in terms of rules and procedures, whereas in the declarative approach, the feature definition is coded in a non-procedural language where a collection of specified constraints are resolved. This makes the procedural approach a better choice for handling specific cases, the declarative approach being suitable for generalization (Shah 93). Aside from being easier to implement, the procedural approach is closer to the structure of the design process. Moreover, it avoids conflicts and allows the use of unidirectional links. The derivation link that we introduce, being unidirectional in nature, can therefore be managed through the procedural approach. However, its applicability is restricted to a specific class of design problems.

The proposed steps are shown in Figure 2. It begins in Step 1 with the identification of the operational context by the user. In Step 2 the user partially models the part which will become the target and positions it in the design context by means of the digital mock-up tools provided by the CAD system. In Step 3 the user creates the derivation link by identifying both the datum and target parts in the design context. During Step 4 the application establishes the relative position and orientation of the datum in the target coordinate system. In Step 5 the application reads from the datum model all the information needed to create the pseudo-imprint. In Step 6 the application derives the pseudo-imprint dependent parameters by applying the design rules that were implemented. Step 7 creates the pseudo-imprint on the target part. Finally, Step 8 maintains the link between the target and datum parts. Whenever a change is made to the datum part, change propagation is achieved by repeating Steps 4 to 7 for each pseudo-imprint involved. 


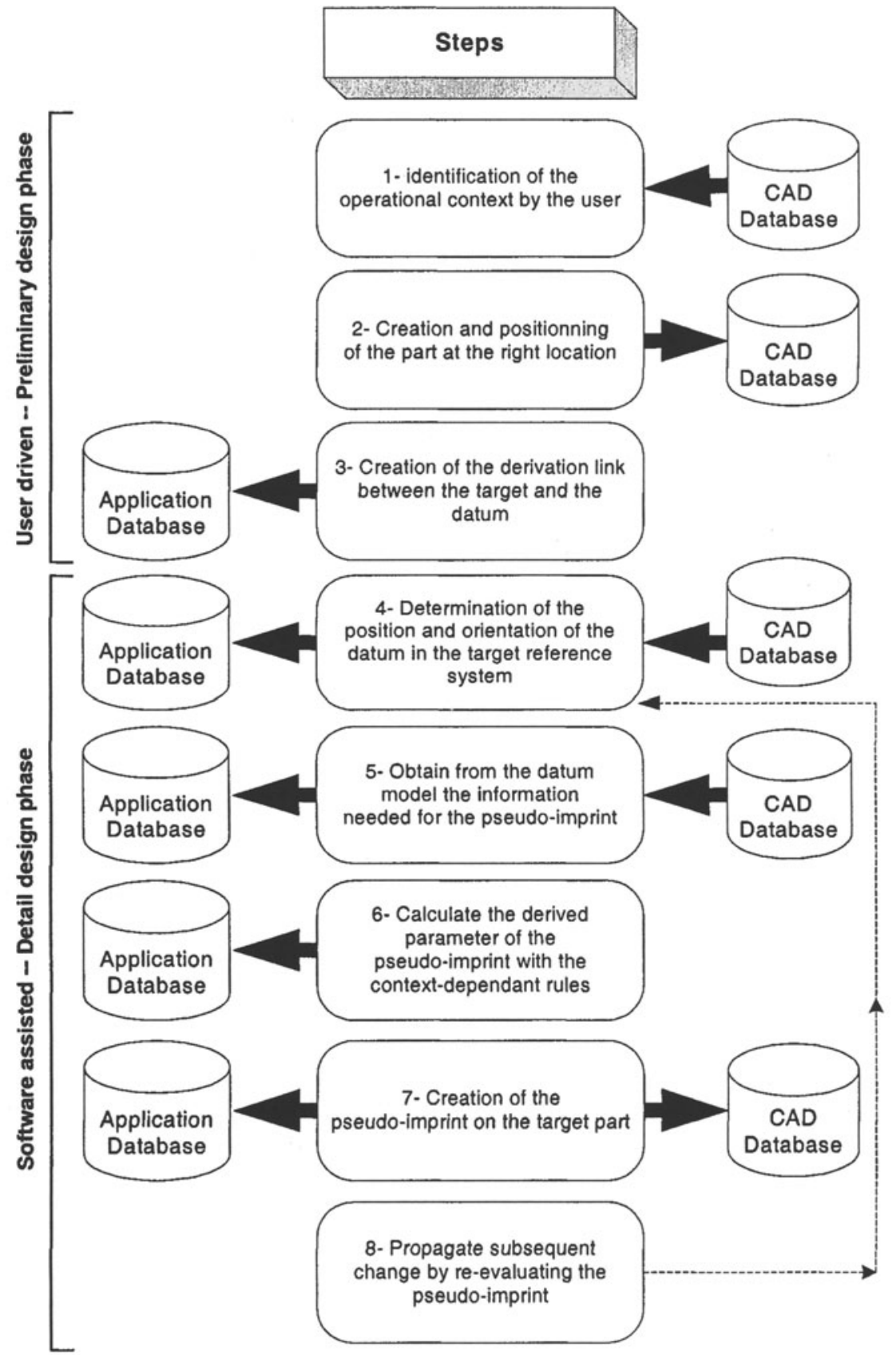

Figure 2. Procedure for the creation of the pseudo-imprint with the derivation link 
The CAD database and application database are shown in Figure 2 to highlight the fact that the application is independent from the CAD platform used. Moreover, because the derivation link is created between features defined by the application rather than by a design-by-feature modeller. Furthermore, Figure 2 shows that this method fits in the design process by capturing, at the preliminary design phase, the information through user interaction which is then used at the detail design phase in the software assisted creation process.

The proposed approach can be used to solve design problems where the geometry of a portion of a part can be derived from the design context and where the specialized technological link can be exploited often enough to justify investing in such dedicated solution. It implies the existence of interfacing features between two parts, for example the keyway in a shaft / key assembly. Also, there must be some sort of hierarchical link between the features of the two parts, so that one acts as reference to the other.

\section{APPLICATION EXAMPLE}

In the aeronautics industry, products are very complex, often made of some tens of thousands of components. This industry recognizes the capability of CAD tools to improve the quality, lead-time and development costs of its products, as evidenced by its systematic use of digital mock-up technology. The application example discussed hereafter was created as a specific project to assist airframe structure designers at Bombardier Aerospace in the modelling of joggles in order to enhance design productivity.

Figure 3 shows a few typical structural parts that can be found in an aircraft. This specific application of technological links focuses on two target features: joggles and cut-outs. The joggle, commonly found in an aircraft's structure, can be defined as a local depression performed on a part to provide an adequate mating area with juxtaposed parts (Figure 4). A cut-out is literally an opening made in a part to accommodate the passing of another part (Figure 5, Step 6). With current CAD systems, manual solid modelling of cut-outs and especially of joggle geometry is a long, repetitive and tedious task. Moreover, an aircraft contains thousands of these features. For cut-outs, the manual definition process generally requires profiles to be created by adding some clearance to the geometry of the design context. The profile is also designed to minimize weight and manufacturing constraints. Once designed, the profile is extruded and the resulting solid is subtracted from the main body. The joggle design process also requires the designer to apply 
knowledge to the design context geometry in order to define construction geometry used to create some solid primitives. These manipulations represent a significant amount of engineering time at the detail design phase, where parts are known to be modified often (the design activity being iterative in its very nature), before being completed and sent to manufacturing. Furthermore, repeated modifications of the models easily lead to product data inconsistencies within the digital mock-up.

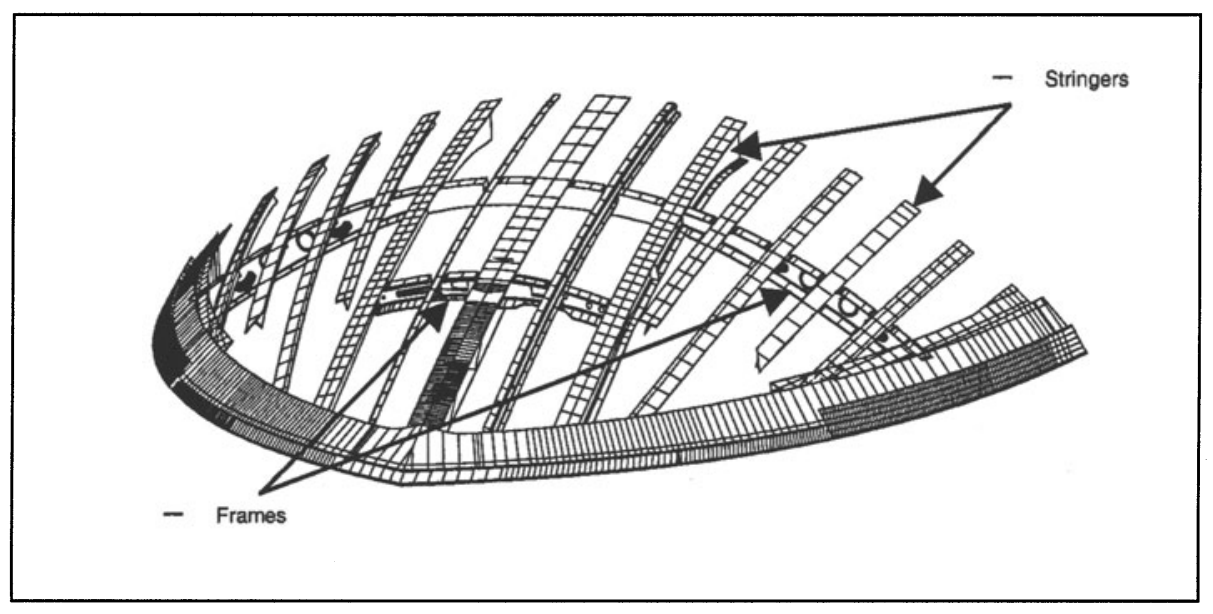

Figure 3. Typical structural parts found in an aircraft

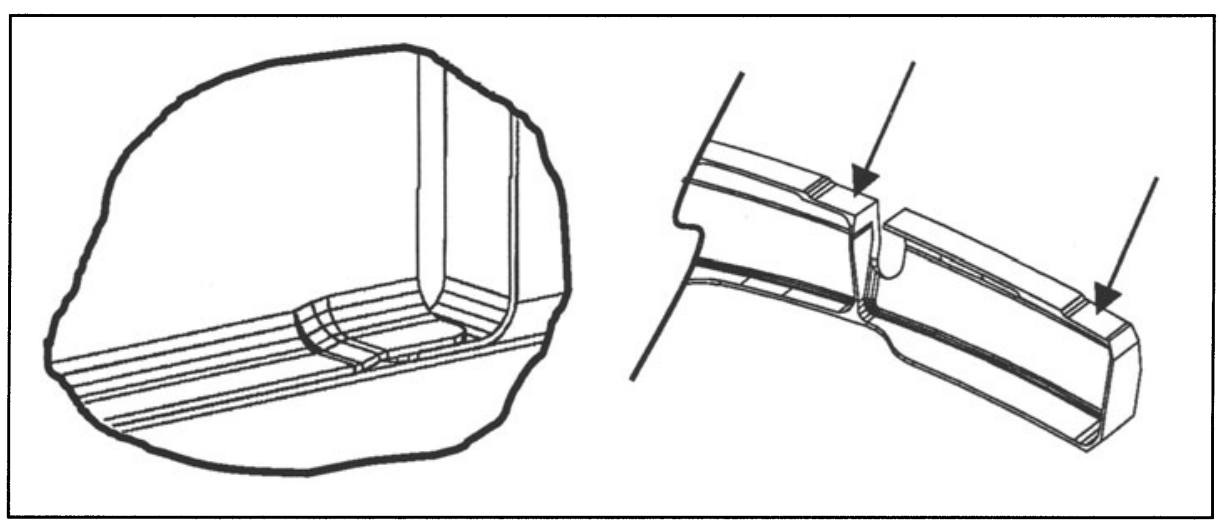

Figure 4. Joggle in a sheet metal part (left) and in a machined part (right) 

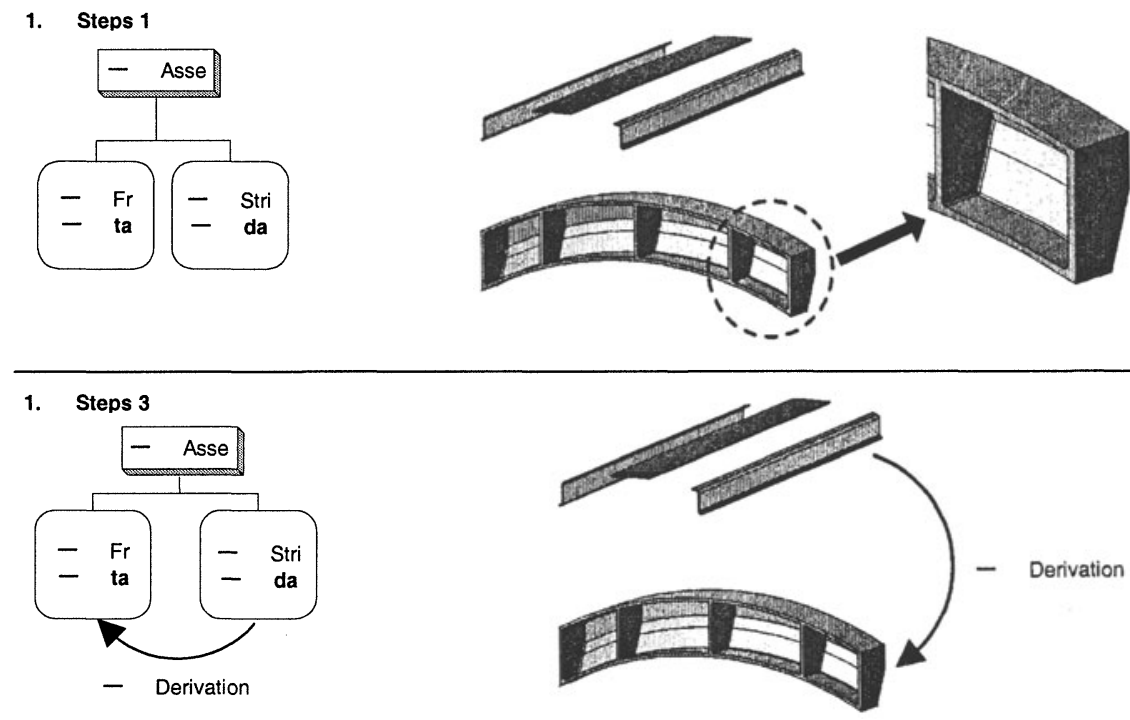

1. St

$\begin{array}{ll}- & \text { rule } 1=\text { offset of cut-out from } \\ - & \text { rule } 2=\text { cut-out } \\ - & \text { rule } 3=\text { joggle } \\ - & \text { rule } 4=\text { joggle }\end{array}$

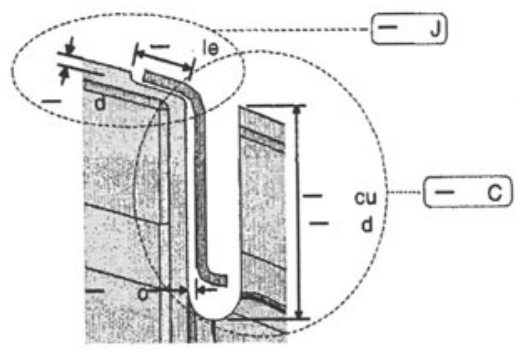

1. Steps 7 and
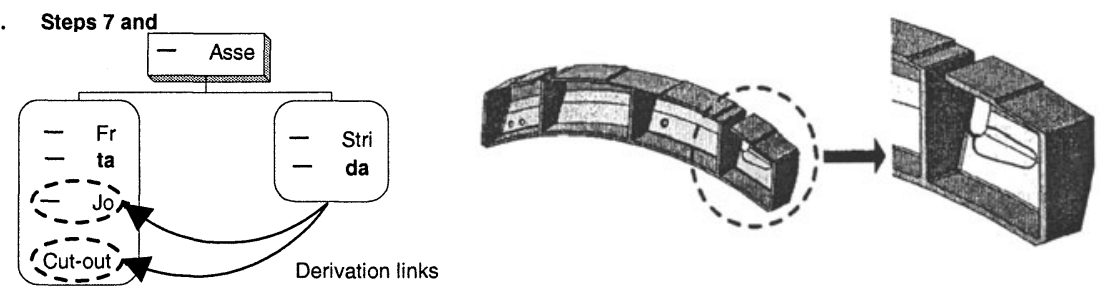

1. Final

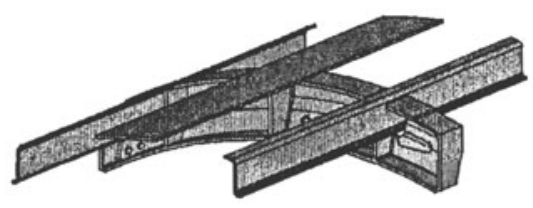

Figure 5 Creation process of a joggle and a cut-out 
The great incidence of this problem and since the shape of joggles is mostly governed by the structural parts involved in the design context, makes it a convincing example for the application of the pseudo-imprint and derivation link concepts along with the method presented previously. Figure 5 illustrates the assisted creation process of a joggle and a cut-out using the proposed method.

In Figure 5 the first stage represents Steps 1 and 2 of the method, where the designer identifies the joggle/cut-out creation problem, creates the basic geometry of the frame and positions this part in the design context. The second stage represents Steps 3 to 5, during which the derivation link is established once the designer has selected the datum part (the stringer) and the target part (the frame) from the design context. With this technological link, the application determines the relative position and orientation of the stringer in the frame coordinate system. Thereafter, it obtains from the stringer model the independent parameters needed to define the joggle and the cut-out. These values are stored with the derivation link in the application database. Subsequently, in Step 6, the geometry of the pseudoimprint is chosen according to the analysis of the geometry of the design context and according to the knowledge implemented. The dimensions of this geometry are then calculated by means of specific design rules. During Step 7 the pseudo-imprints are created on the frame, thereby forming the joggle and the cut-out. Finally, Step 8 involves the propagation of all subsequent changes for pseudo-imprint re-evaluation, thus ensuring that the coherence of the assembly is maintained. The latter step is performed when the geometry of the stringer or the position of one of the two parts has changed.

\section{CONCLUSION}

The proposed framework brings many benefits to some of the modelling tasks found in the design process. Firstly, it provides a net increase in productivity for specific, long, repetitive and tedious modelling tasks. This is achieved in part by allowing designers to interact with the CAD system at a higher semantic level to capture design intent. Secondly, it enables the formalization of design knowledge stored in the application database, such function being triggered by technological links. Lastly, the proposed framework makes for a CAD system that is highly flexible and reactive to modifications in product definition, one of the keys to maintaining product data consistency in the digital mock-up.

In summary, our method exploits the design context by creating a derivation link between two parts in order to obtain specific information 
from the datum part. Such information is then used to derive the pseudoimprint by applying specific design rules previously implemented in the application. Subsequently, the pseudo-imprint is created on the target part, and the derivation link is maintained to keep the coherence of the assembly. In so doing, we reach our objective of exploiting the design context to manage product data consistency. The proposed solution is CAD-system independent and uses the top-down approach so as to support the design process from the preliminary to the detailed design phase.

In contrast to generic modelling tools, the proposed dedicated applications make use of specific knowledge to relieve designers from performing tedious tasks, thereby enhancing their productivity. Since dedicated applications are associated to specific design problems, the proposed framework was applied to a specific design task. However, the basic concepts are generic enough to be applied to a collection of other design problems by defining different technological links to capture relationships between two or more parts. More specifically, the proposed approach can be applied whenever repetitive context-dependant geometry need to be defined on parts or features sharing specific common parameters and attributes (therefore constituting a "class" in terms of oriented object programming). As a result, the proposed framework offers strong potential for the development of specific task oriented applications to help designers solve various design problems.

\section{ACKNOWLEDGEMENTS}

The work reported in this article was supported by the NSERC (the Natural Sciences and Engineering Research Council of Canada), by the J.A. Bombardier Foundation and by the École de Technologie Supérieure.

\section{REFERENCES}

Bordegoni, M., and Cugini, U. (1999). Feature-based assembly in aeronautics design: from concepts development to formalization, International Journal of Vehicle Design, V.21, No.2, pp.228-242.

Bronsvoort, F., and Jansen, F.W. (1994). A multi-view feature modeling for design and assembly, Advances in Feature Base Manufacturing, J.J. Shah, M. Mantyla and D.S. Nau, Elsevier Science B.M.

Deneux, D. (1999). Introduction to assembly features - an illustrated synthesis methodology, Journal of Intelligent Manufacturing, Kluwer Academic Publishers, V.10, No.1, pp.29-39. 
Gui, J.-K., and Mäntylä, M. (1993). New concepts for complete product assembly modeling, Proceedings of the 2nd Symposium on Solid Modeling and Applications, May 19-21, pp.397-406.

Gui, J.-K., and Mäntylä, M. (1994). Functional understanding of assembly modeling, Computer Aided Design, V.26, No.6, pp.435-451.

Libardi, E.C., Dixon, J.R., and Simmons, M.K. (1988). Computer environments for the design of mechanical assemblies: a research review, Eng. Comput., pp.121-135.

Macabies, L., Desrochers, A., Rivest, L., and Maranzana, R. (2000). Liens multi-modèles en CAO - Application au rivetage en aéronautique, 3rd International Conference on Integrated Design and Manufacturing in Mechanical Engineering Forum 2000, Canadian Society for Mechanical Engineering, May 17-19, Montreal, Canada, Paper No 271.

Mailharro, D. (1998). A classification and constraint-based frame-work for configuration, Artificial Intelligence for Engineering Design, Analysis and Manufacturing, V.12, pp.383397.

Rimscha, M.V. (1990). Feature modeling and assembly modeling - A unified approach, Proceedings of the IFIP WG 5.2/GI International Symposium, Nov 8-10, pp.203-213.

Shah, J.J., and Mäntylä, M. (1995). Parametric and Feature-Based CAD/CAM, John Wiley \& sons, pp.173-186.

Shah, J.J., and Tadepalli, R. (1992). Feature based assembly modeling, Proceedings of the 1992 ASME International Computers in Engineering Conference and Exposition, Aug 2-6, pp.253-260.

Shah, J.J., and Rogers, M.T. (1993). Assembly modeling as an extension of Feature-Based Design, Research in Engineering Design, V.5, pp.218-237.

Sodhi, R. (1991). Representing tolerance and assembly information in a feature based design environment, Advances in design Automation, V.1, pp.101-108.

Ullman, D.G. (1997). The mechanical design process, 2nd edition, The McGraw-Hill Companies, Inc. 